\title{
Salud mental y calidad de vida en adultos mayores: revisión sistémica
}

\section{Mental health and quality of life in older adults: systemic review}

Marcela Gálvez Olivares ${ }^{1}$, Cristopher Aravena Monsalvez ${ }^{1}$, Hernan Aranda Pincheira ${ }^{1}$ Carolina Ávalos Fredes ${ }^{1}$, Fanny López-Alegría ${ }^{2}$

\begin{abstract}
Introduction: Currently the number of people over 60 years of age is the fastest growing age segment worldwide, which represents a great challenge for public health. Objective: Identify through scientific evidence, the existence of a relationship between mental health disorders and the quality of life of older adults. Method: The research was carried out through a systematic review, in January 2020, in the bibliographic databases MEDLINE, LILACS, CINAHL and SCIELO, using the descriptors "aged", "elderly", "aged, 80 and over", "mental health"," Dementia ", "depression ", "alzheimer disease "," quality of life "," WHOQOL-BREE "," WHOQOL-BREF "," WHOQOLOLD ", "SF-12 ", "SF-36 ", "NdF", "geriatric depression scale" and "mental health scales". Results: Of the 1,231 articles located, 15 were included in the analysis. A statistical association between the risk of depression and impaired quality of life was demonstrated in 4 studies. However, a study showed that the population of older adults had a quality of life defined as medium or high, despite $31.1 \%$ of this population having mental disorders such as stress, depression or anxiety. Conclusions: To assess the quality of life and mental health, an increase in publications in the last 3 years is evident. The instruments used were various scales or questionnaires. Several factors that positively or negatively affect the relationship between mental health and quality of life of older adults were identified.
\end{abstract}

Key words: Aged; Mental Health; Quality of Life Rev Chil Neuro-Psiquiat 2020; 58 (4): 384-399

\section{Introducción}

E Informe Mundial sobre Envejecimiento y Salud desarrollado por la Organización Mundial de la Salud (OMS) presenta- do en 2015, refiere que a nivel mundial el número de personas con más de 60 años se duplicará para el año 20501. Este rápido aumento de la población de adultos mayores, que conlleva una mayor longevidad, depen-

Los autores declaran no tener conflictos de interés.

Aceptado: 2020/08/06

Recibido: 2020/03/24

Alumno de la Univ. Andrés Bello, Fac. Enfermería, Escuela de Enfermería, Santiago, Chile. Matrona, Doctor en Salud pública, Docente de la Univ. Andrés Bello, Fac. Enfermería, Santiago, Chile. 
de de un factor clave: que es la salud en todos sus aspectos, incluida la salud mental. A nivel mundial, aproximadamente un 15\% de los adultos mayores padecen algún tipo de trastorno mental, siendo la demencia, la depresión y el síndrome confusional agudo los trastornos más comunes 1-4.

La OMS reconoce que no existe una definición oficial de salud mental, ya que las definiciones varían de acuerdo a determinadas diferencias culturales, presupuestos subjetivos y teorías profesionales, pero preconiza la que se refiere al "bienestar subjetivo, autonomía, competencia, dependencia intergeneracional y reconocimiento de la habilidad de realizarse intelectual y emocionalmente" 1,2. Al no existir una definición oficial, se considera la de la Asociación Guipuzcoana de Familiares y Personas con Problemas de Salud Mental (AGIFES), que establece que salud mental es el "estado de equilibrio emocional, cognitivo y conductual que permite al individuo desenvolverse de manera responsable en su entorno familiar, social y laboral, así como gozar de bienestar y calidad de vida" 5. Esta última definición está más vinculada con el mejoramiento de la calidad de vida y el potencial para ganar una mejor salud, que solo con una disminución de síntomas6.

Así, calidad de vida, ha sido definida por la OMS como "la percepción del individuo de su posición en la vida en el contexto de la cultura y los sistemas de valores en los que vive y en relación con sus objetivos, expectativas, estándares y preocupaciones" 7. De esta forma, al vincular el concepto de calidad de vida con el de salud emerge la definición de Urzúa, quien considera este ámbito "como el nivel de bienestar derivado de la evaluación que la persona realiza de diversos dominios de su vida, considerando el impacto que en éstos tiene su estado de salud" 8 .

Por tanto, de acuerdo a este contexto y a las posibles repercusiones en la vida cotidia- na de los adultos mayores, se establece como objetivo, identificar a través de evidencias científicas, la existencia de una relación entre los problemas o trastornos de salud mental y la calidad de vida de este grupo etario.

\section{Metodología}

Se realizó una revisión sistemática de la literatura relacionada con el tema trastornos de salud mental y calidad de vida de los adultos mayores, de acuerdo a las etapas recomendadas por la Colaboración Cochrane, que son: formulación de la pregunta de investigación, establecimiento de los criterios de inclusión y exclusión, localización de los artículos, aplicación de criterios de calidad metodológica, recolección de datos, análisis y presentación de los resultados 9,10

Esta revisión sistemática fue guiada por la siguiente pregunta de investigación: ¿existen evidencias científicas que identifiquen la presencia de una relación entre los problemas o trastornos de salud mental y la calidad de vida de los adultos mayores? Esta pregunta es sustentada en la estrategia PICO con los siguientes componentes: Paciente adultos mayores y ancianos (65 y más años); Intervención - conductas o factores que intervienen en la salud mental que mejoran la calidad de vida; Comparación - problemas o patologías que intervienen en la salud mental que deterioran la calidad de vida y "Outcome" - niveles de calidad de vida asociados a patologías o trastornos de la salud mental como depresión, demencia senil o mal de Alzheimer.

Para esta estrategia de búsqueda se utilizaron las bases de datos Medical Literature Analysis and Retrieval System online (MEDLINE), Cumulative Index to Nursing and Allied Health Literature (CINAHL), Literatura Latino-Americana e do Caribe em Ciencias da Saúde (LILACS) y Scientific Electronic Library Online (SciELO), consultadas en el mes de enero de 2020. 
En esta búsqueda se utilizaron descriptores seleccionados en inglés del Medical Subject Headings (MeSH), en español y portugués de los Descriptores en Ciencias de la Salud (DeCS) junto a los operadores booleanos (AND y OR). Los elementos anteriores fueron utilizados para la siguiente estrategia de búsqueda en MedLine y luego se adaptó a las otras bases de datos: Search $((()(($ Aged $)$ OR Elderly) OR (Aged, 80 and over))) AND ((((mental health) OR dementia) OR depression) OR alzheimer disease)) AND quality of life) AND ((()(WHOQOL-BREE) OR WHOQOL-BREF) OR WHOQOL-OLD) OR SF-12) OR SF-36)) AND (((Geriatric depression scale) OR mental health scales) OR NdF)

Luego a esta búsqueda electrónica, se aplicaron los criterios de inclusión y exclusión. Los criterios de inclusión fueron: artículos de investigación, revisiones sistémicas, metaanálisis y guías clínicas publicadas entre los periodos 2009 a 2019 en español, inglés y portugués. Entre las características particulares del sujeto en estudio se incluyen a adultos mayores y ancianos (65 y más años) con salud o enfermedad mental. Los criterios de exclusión fueron: revisiones narrativas, cartas al editor, artículos de opinión, relatos de experiencia, casos clínicos, libros, presentaciones en congresos, estudios en los que no se apreciaba con claridad el método de medición de la variable en estudio y artículos que no respondieran a la pregunta de investigación. Entre las características particulares, se excluyeron a los adultos mayores y ancianos institucionalizados; además de los que estaban con tratamiento de enfermedades mentales.

Una vez identificados los artículos en las bases de datos, se procedió a la eliminación de los registros duplicados mediante el software EndNoteBasic, Thomson Reuters, USA. A continuación, para refinar más la estrategia de búsqueda se procedió a ejecutar 2 fases de eliminación de registros. La prime- ra, se realizó mediante la lectura de títulos y resumen y la segunda, a través de la lectura a texto completo. A este número de artículos seleccionados a texto completo, se aplicaron los criterios de evaluación de calidad metodológica de los estudios mediante la escala AMSTAR 2005 (Assrssment of Multiple Systematic Reviews) para las revisiones sistemáticas y para los estudios observacionales, la lista Strenghening the Reporting of Observational Studies in Epidemiology (STROBE). Los artículos que obtuvieron una puntuación inferior a $80 \%$ fueron eliminados, obteniéndose en esta última fase los artículos incluidos para revisión. Todo este proceso de identificación, selección y evaluación de la elegibilidad de los artículos se hizo mediante la utilización del protocolo Preferred Reporting Items for Systematic Reviews and Meta-Analyse (PRISMA) 11

El análisis de los artículos seleccionados fue realizado por 3 autores de forma independiente y las divergencias fueron resueltas a través de un consenso después de una nueva revisión del o los artículos en discordancias. Este análisis fue hecho mediante una revisión descriptiva y analítica de los resultados encontrados en la lectura de los artículos a texto completo. Estos resultados fueron presentados y evaluados en cuanto a su aplicabilidad y/o respuesta a la pregunta de investigación en un cuadro sinóptico que considera los siguientes aspectos: identificación del artículo, país de realización, año del estudio, tamaño de la muestra, edad de los adultos mayores, tipo de diseño, características mentales de los participantes al ingreso al estudio, escalas de evaluación, relación entre salud mental y calidad de vida y el aporte del estudio a la pregunta de investigación (Tablas 1 y 2).

Respecto a los aspectos éticos, fue respetada la integridad de los artículos, es decir, no se realizaron modificaciones de los contenidos encontrados. 
Tabla 1. Caracterización de los estudios de salud mental y calidad de vida de los adultos mayores, Santiago, Chile, 2020

\begin{tabular}{|c|c|c|c|c|c|c|c|}
\hline 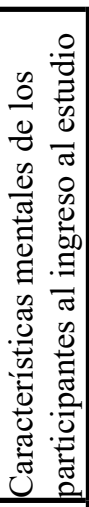 & 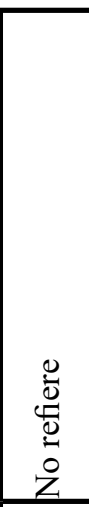 & 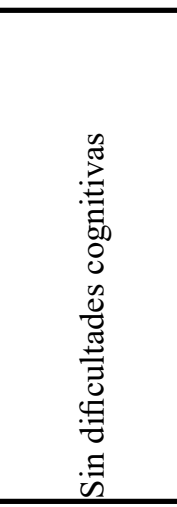 & $\begin{array}{l}0 \\
\stackrel{0}{0} \\
\stackrel{0}{0} \\
: \\
0 \\
Z \\
\end{array}$ & 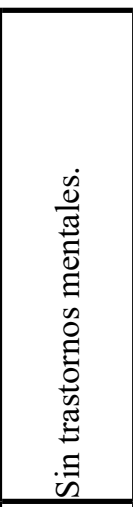 & $\begin{array}{l}0 \\
\stackrel{0}{0} \\
\stackrel{0}{0} \\
: \\
0 \\
Z\end{array}$ & 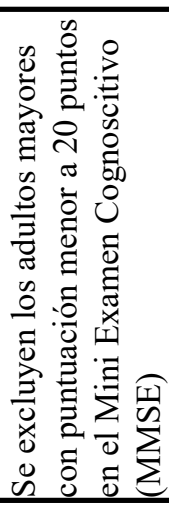 & 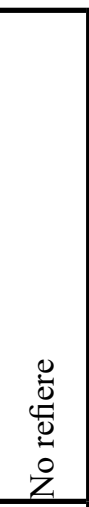 \\
\hline 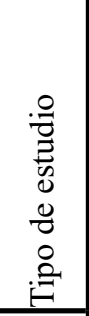 & 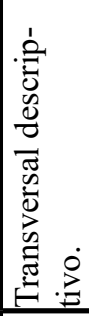 & 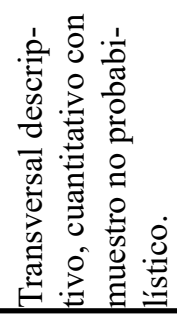 & 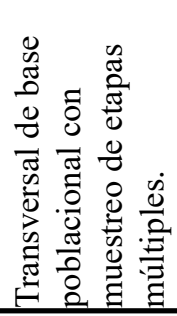 & 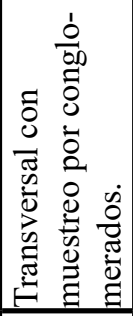 & 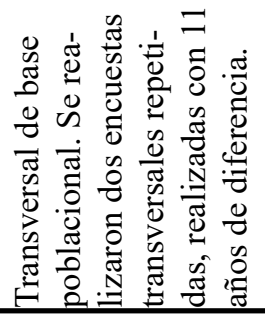 & 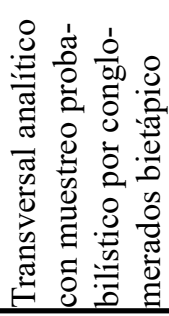 & 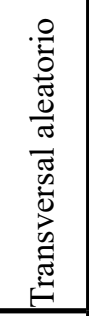 \\
\hline 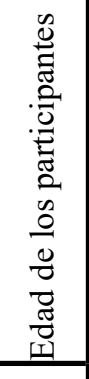 & 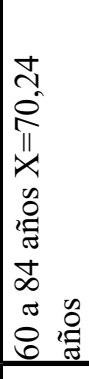 & 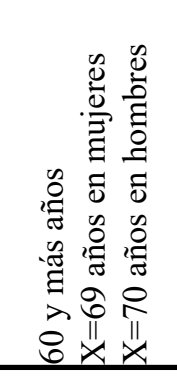 & 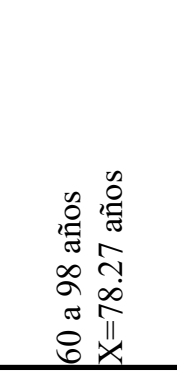 & 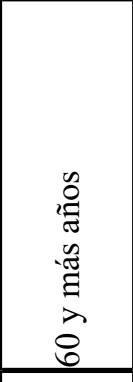 & 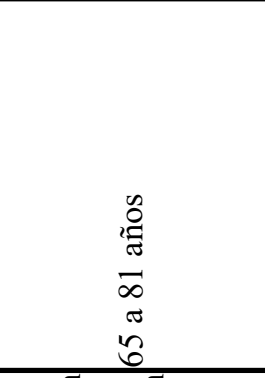 & 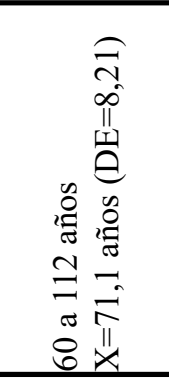 & 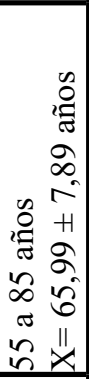 \\
\hline 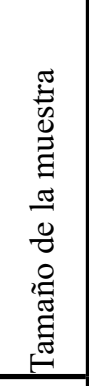 & 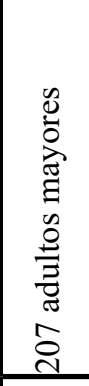 & 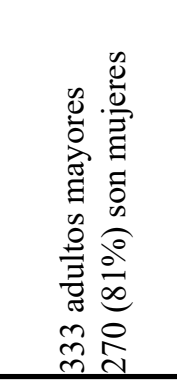 & 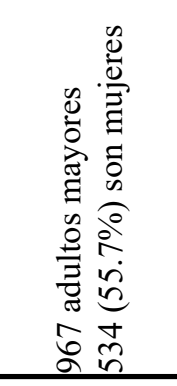 & 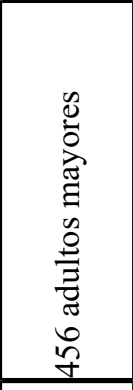 & 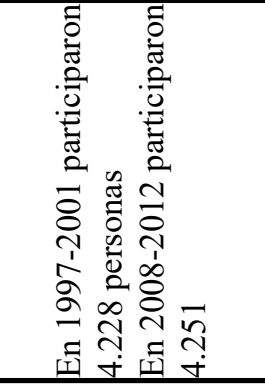 & 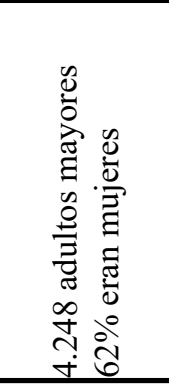 & 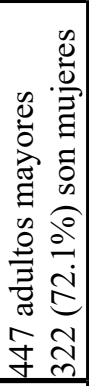 \\
\hline 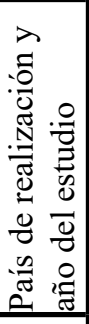 & 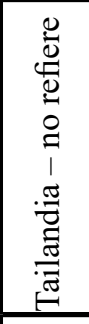 & 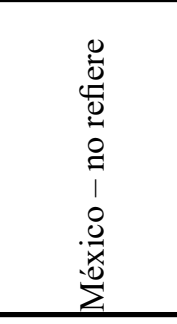 & 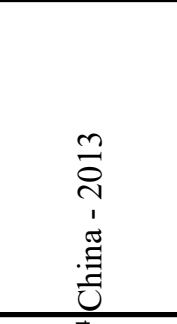 & 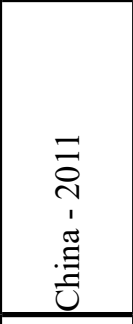 & 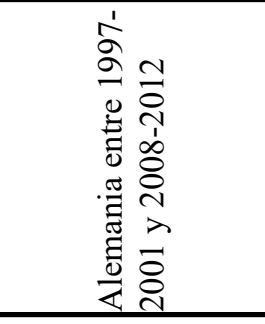 & $\begin{array}{l}1 \\
0 \\
1 \\
1 \\
-\frac{\pi}{0} \\
0 \\
0 \\
0 \\
0\end{array}$ & 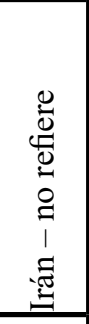 \\
\hline 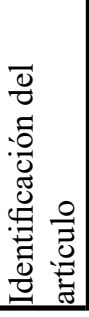 & 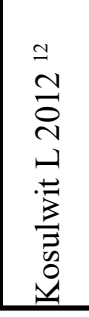 & 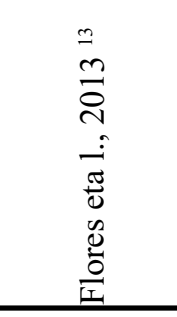 & 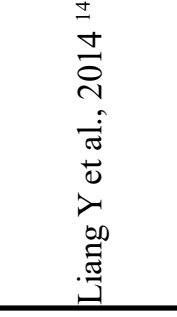 & 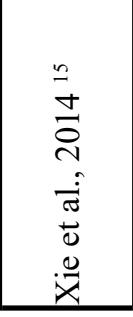 & 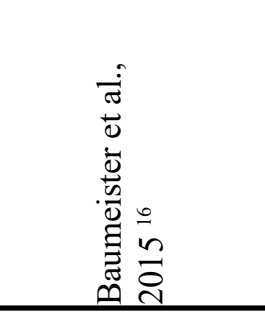 & 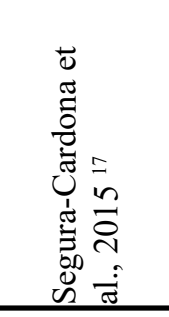 & 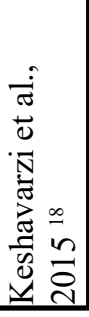 \\
\hline
\end{tabular}


Tabla 1. Caracterización de los estudios de salud mental y calidad de vida de los adultos mayores, Santiago, Chile, 2020. Continuación

\begin{tabular}{|c|c|c|c|c|c|c|c|c|}
\hline 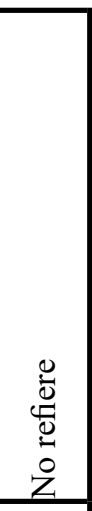 & 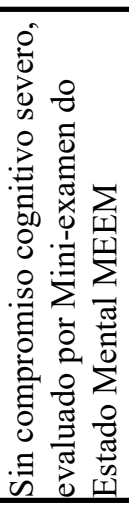 & & 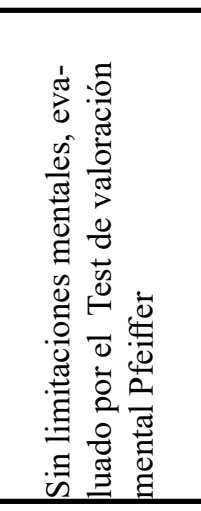 & 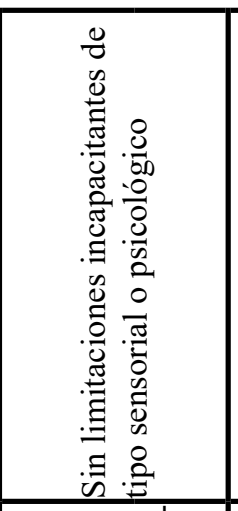 & 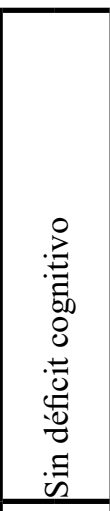 & 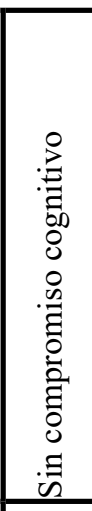 & 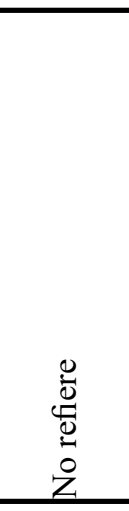 & 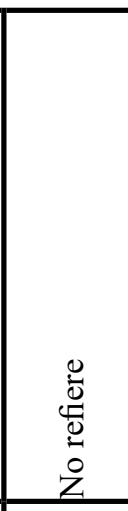 \\
\hline 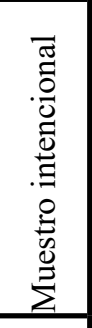 & 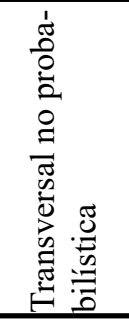 & & 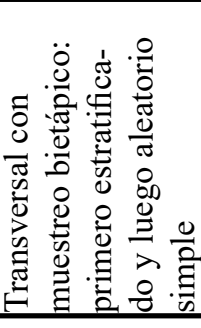 & 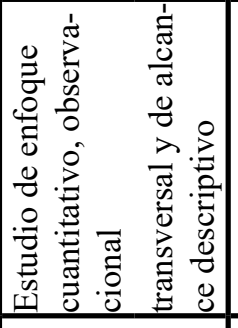 & 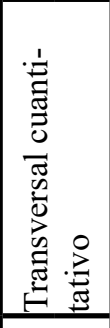 & 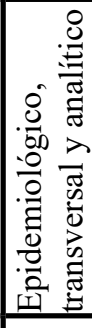 & 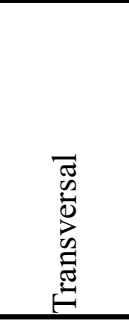 & 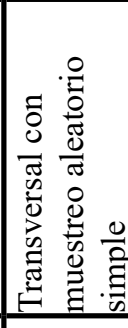 \\
\hline 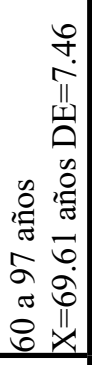 & 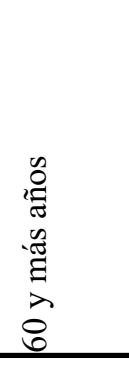 & 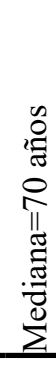 & 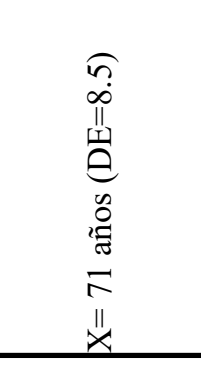 & 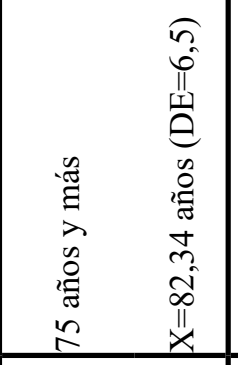 & 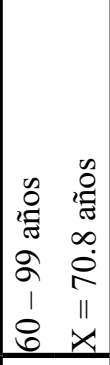 & 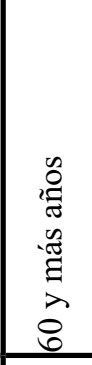 & 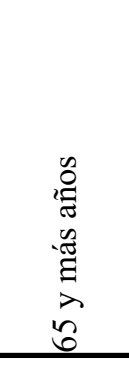 & 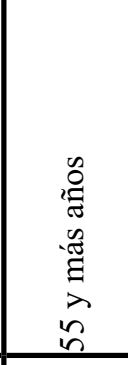 \\
\hline 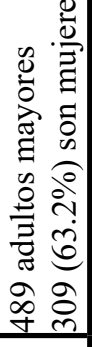 & 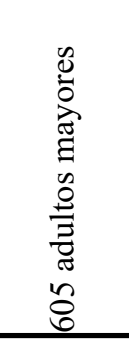 & & 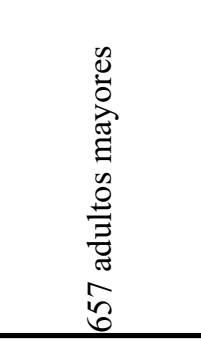 & 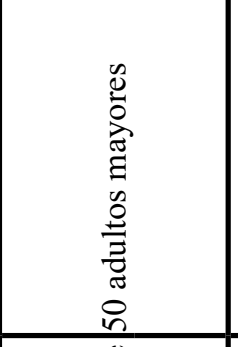 & 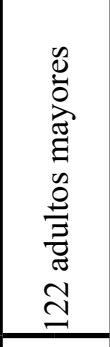 & 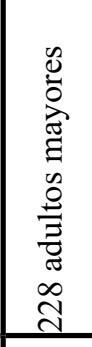 & 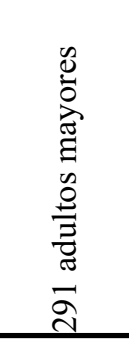 & 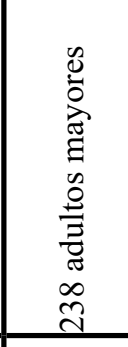 \\
\hline 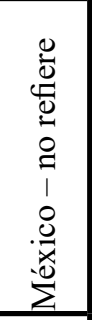 & 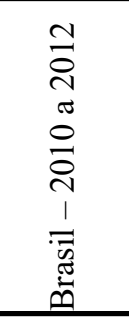 & & 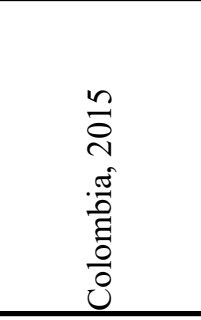 & 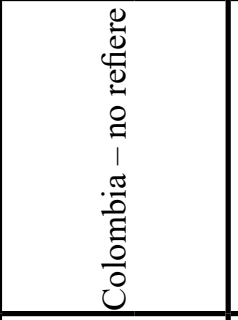 & 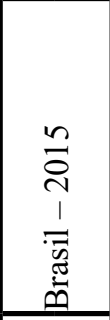 & 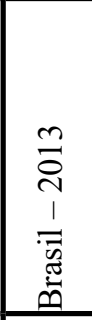 & 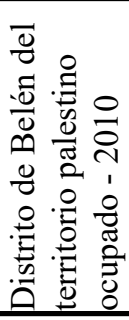 & 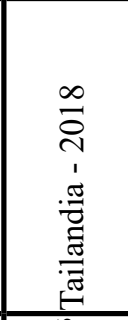 \\
\hline 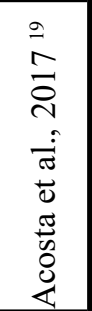 & 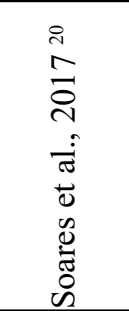 & & 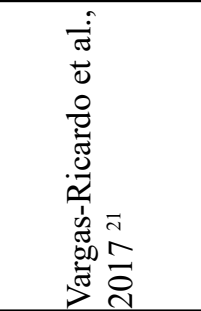 & 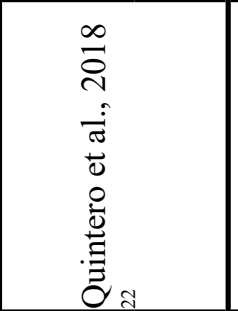 & $\begin{array}{l}2 \\
\infty \\
0 \\
0 \\
i \\
\dot{\pi} \\
0 \\
0 \\
0 \\
0 \\
0 \\
0\end{array}$ & 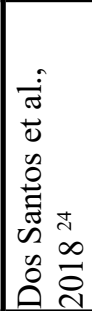 & 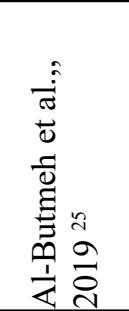 & 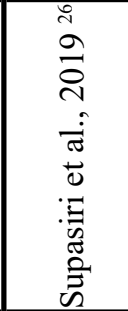 \\
\hline
\end{tabular}


Tabla 2. Escalas de evaluación de la salud mental y calidad de vida de los adultos mayores, resultados de los estudios y aportes a la pregunta de investigación, Santiago, Chile, 2020

\begin{tabular}{|c|c|c|c|c|}
\hline 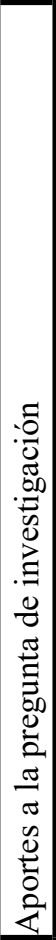 & 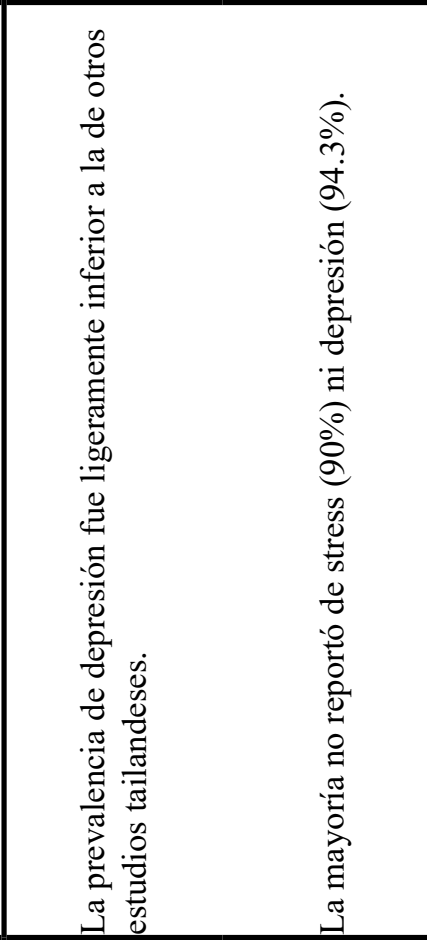 & 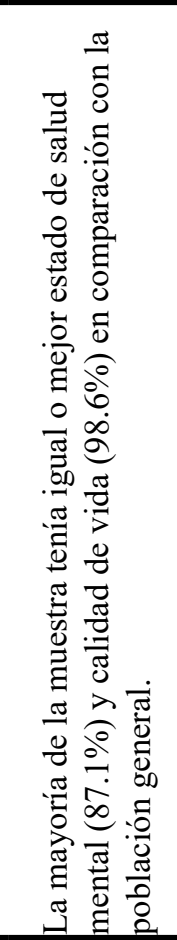 & 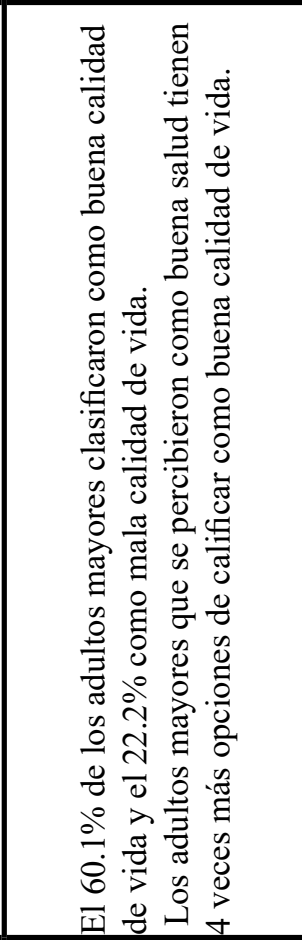 & 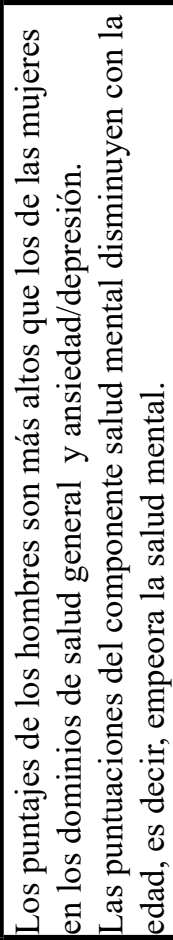 \\
\hline 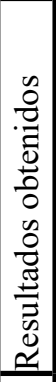 & 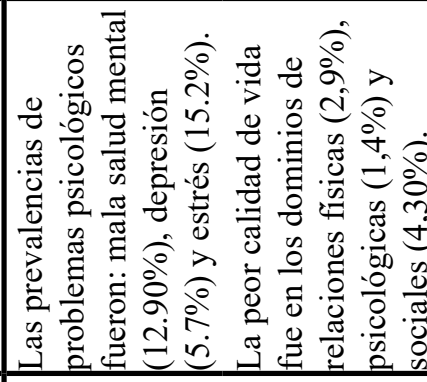 & & 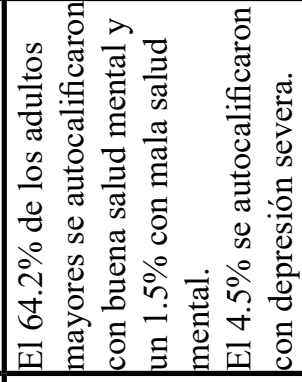 & 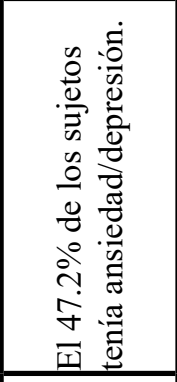 \\
\hline 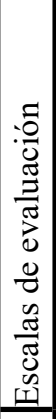 & 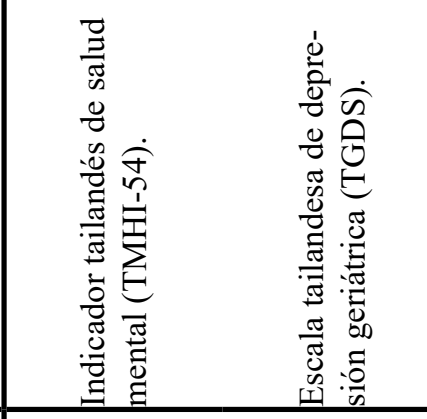 & 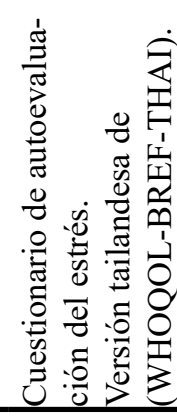 & 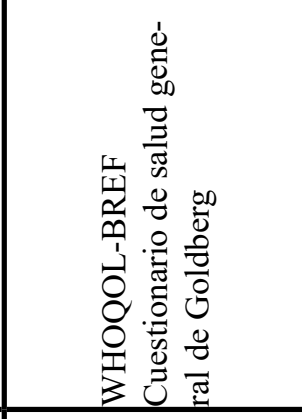 & 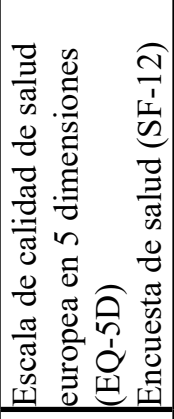 \\
\hline 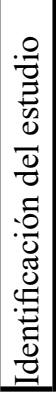 & 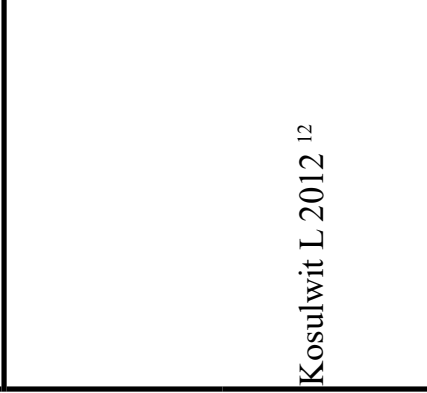 & & 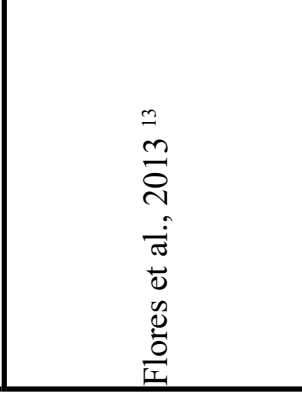 & 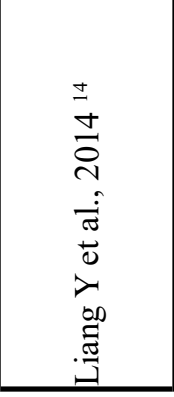 \\
\hline
\end{tabular}


Tabla 2. Escalas de evaluación de la salud mental y calidad de vida de los adultos mayores, resultados de los estudios y aportes a la pregunta de investigación, Santiago, Chile, 2020. Continuacion 1

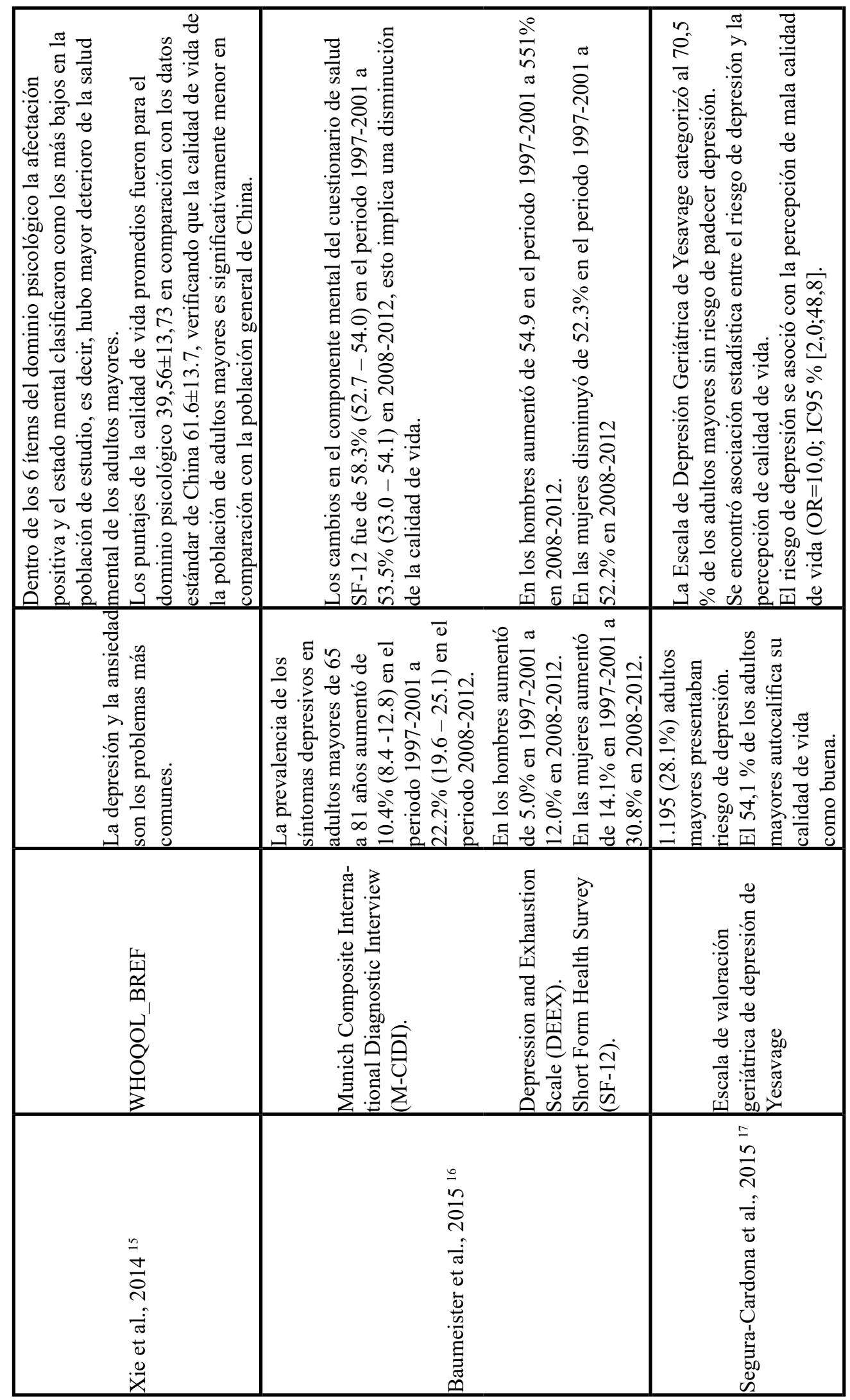


Tabla 2. Escalas de evaluación de la salud mental y calidad de vida de los adultos mayores, resultados de los estudios y aportes a la pregunta de investigación, Santiago, Chile, 2020. Cont. 2

\begin{tabular}{|c|c|c|}
\hline 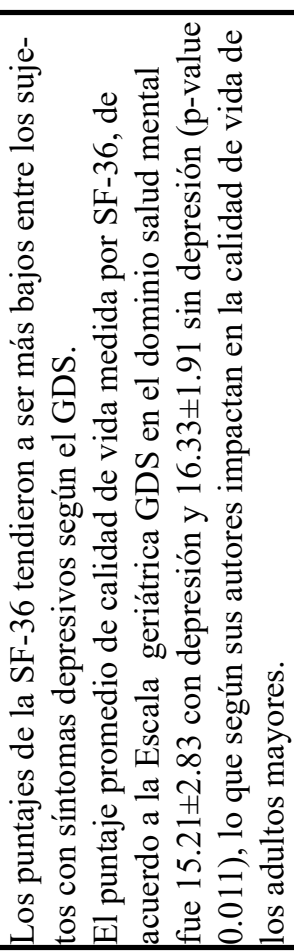 & 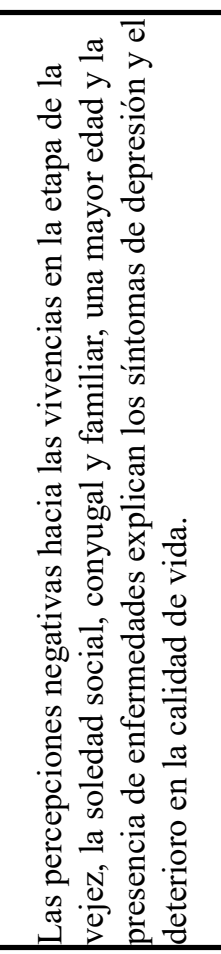 & 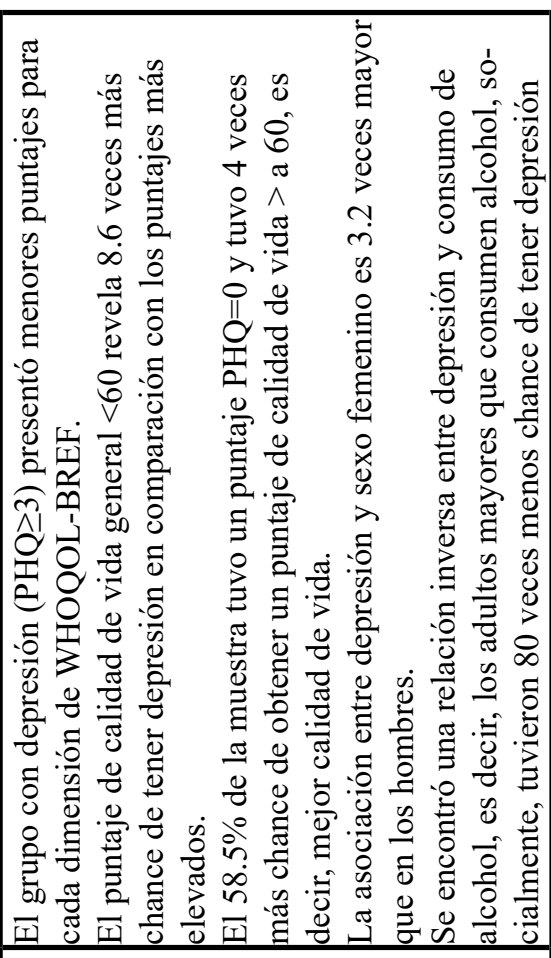 \\
\hline 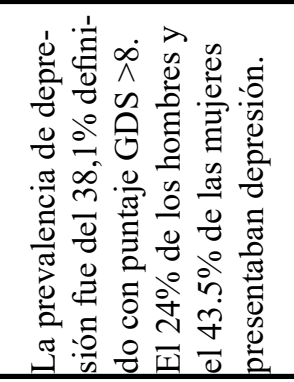 & & 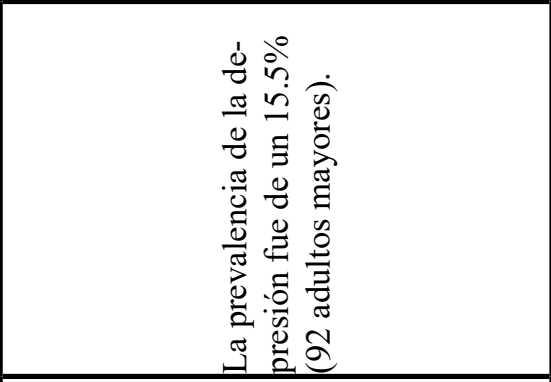 \\
\hline 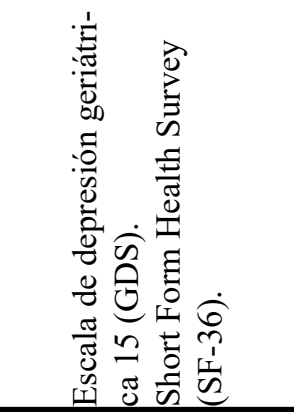 & 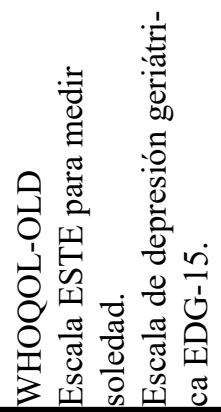 & 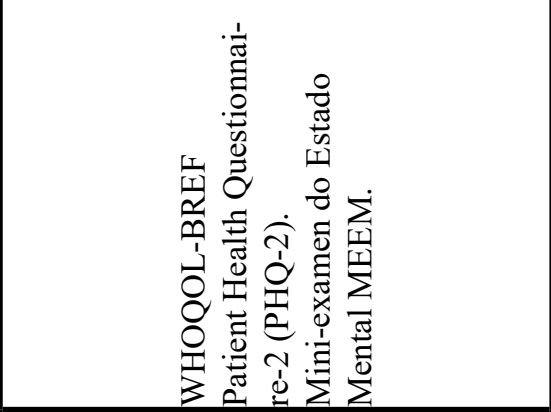 \\
\hline 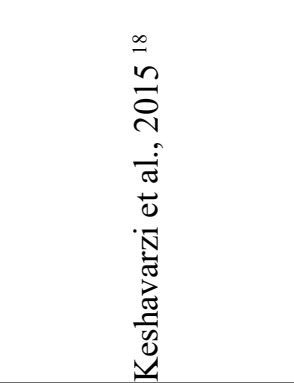 & 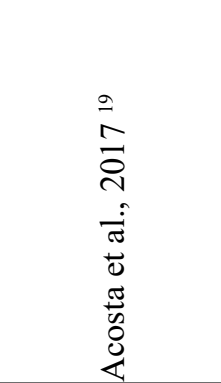 & 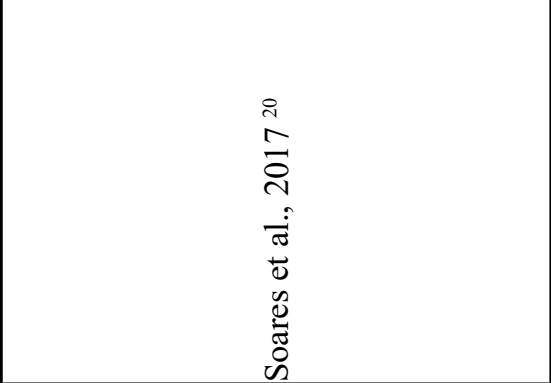 \\
\hline
\end{tabular}


Tabla 2. Escalas de evaluación de la salud mental y calidad de vida de los adultos mayores, resultados de los estudios y aportes a la pregunta de investigación, Santiago, Chile, 2020. Cont.3

\begin{tabular}{|c|c|c|}
\hline 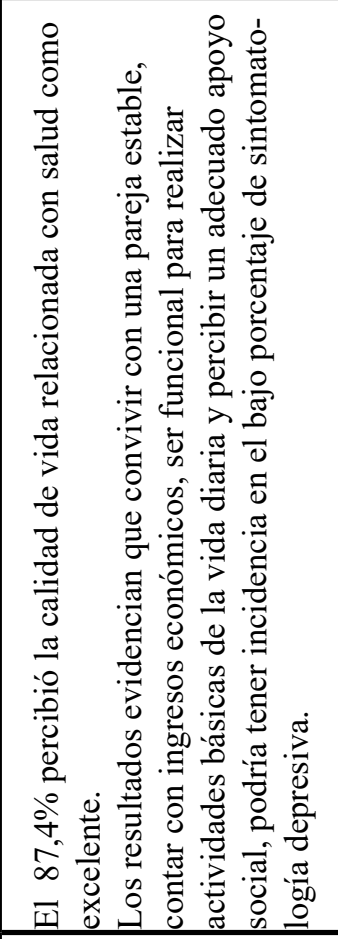 & 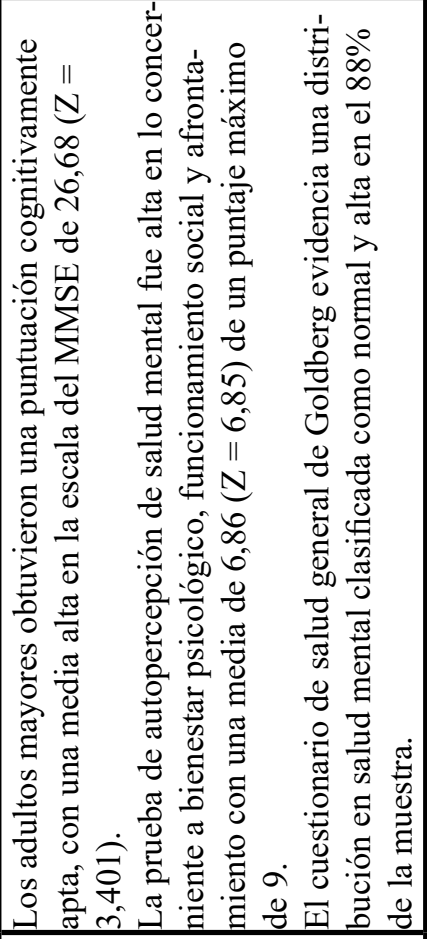 & 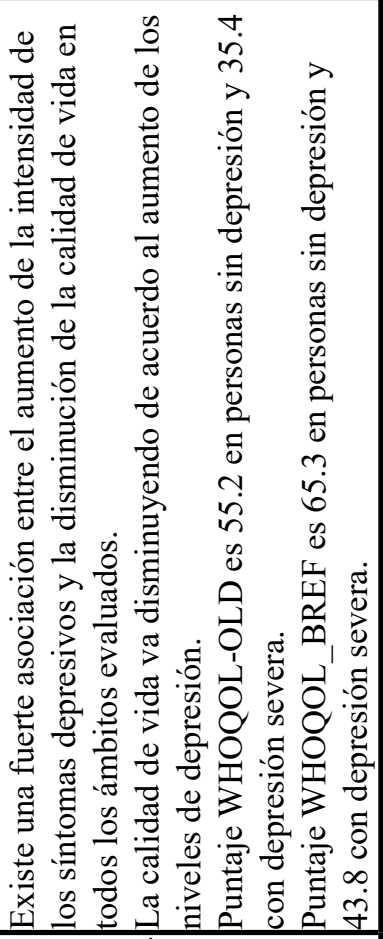 \\
\hline 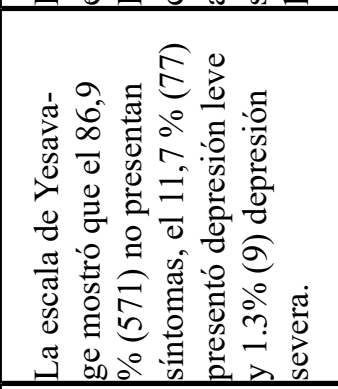 & & 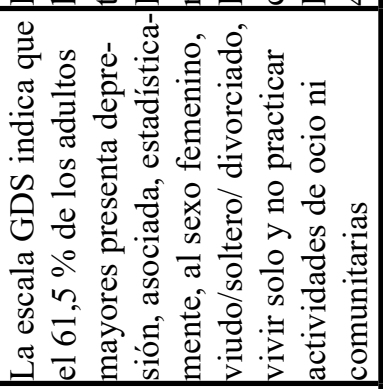 \\
\hline 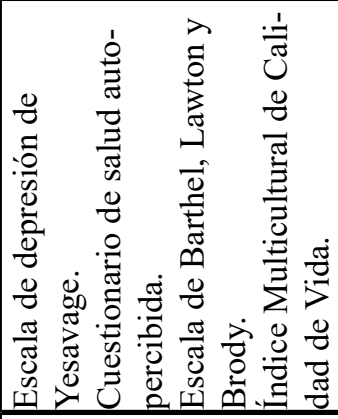 & 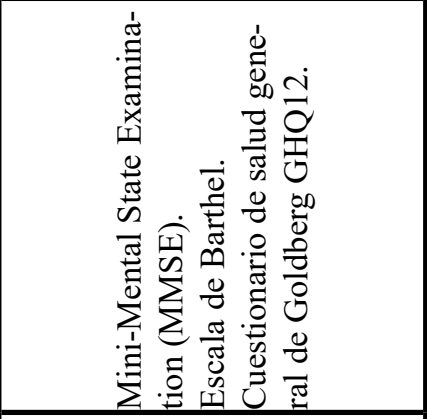 & 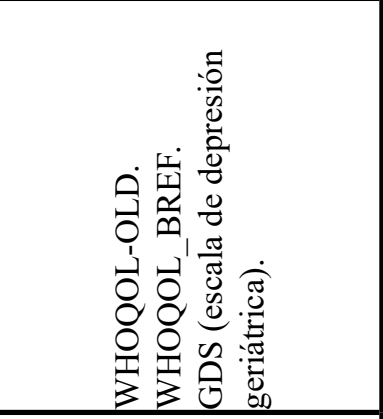 \\
\hline 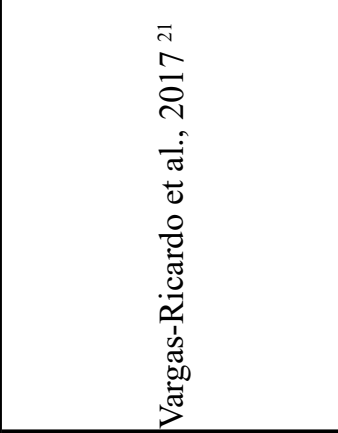 & 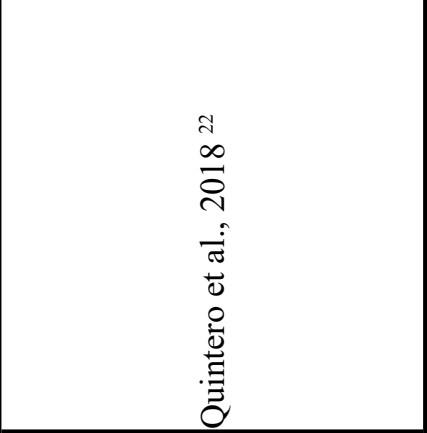 & 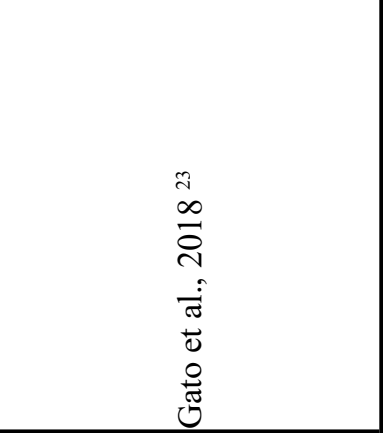 \\
\hline
\end{tabular}


Tabla 2. Escalas de evaluación de la salud mental y calidad de vida de los adultos mayores, resultados de los estudios y aportes a la pregunta de investigación, Santiago, Chile, 2020. Cont.4

\begin{tabular}{|c|c|c|}
\hline 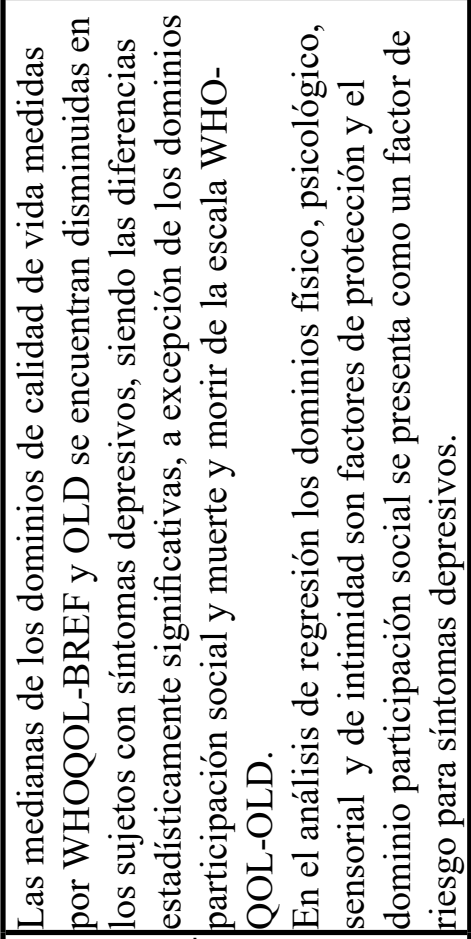 & 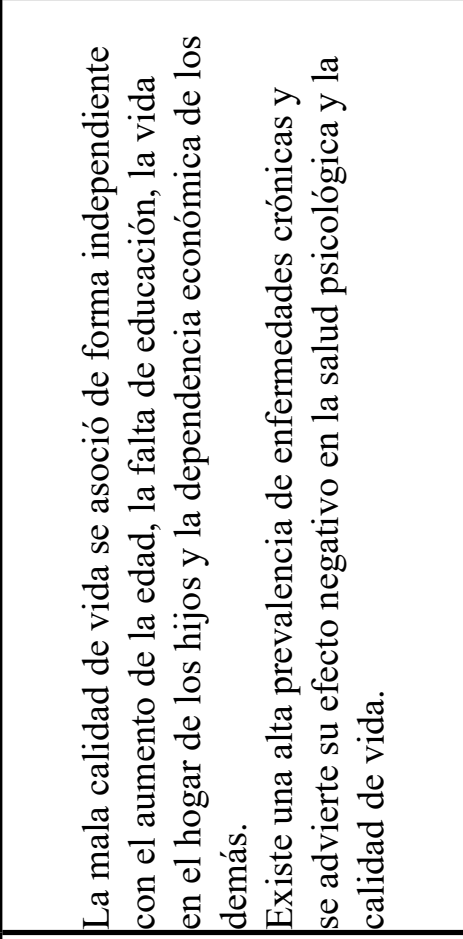 & 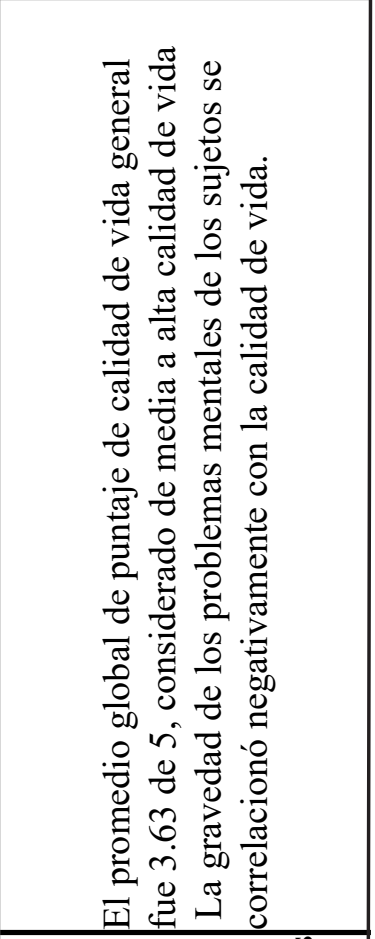 \\
\hline 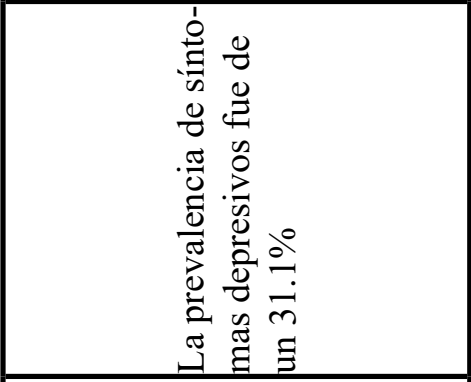 & 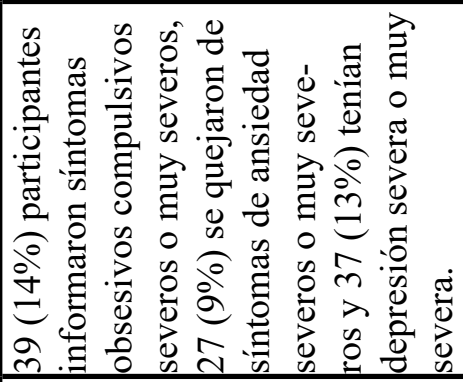 & 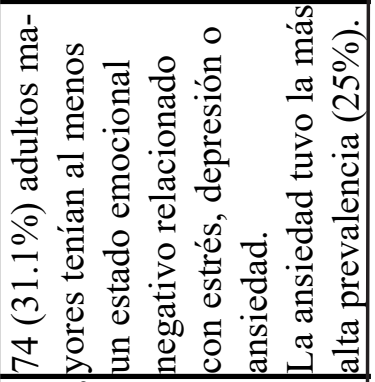 \\
\hline 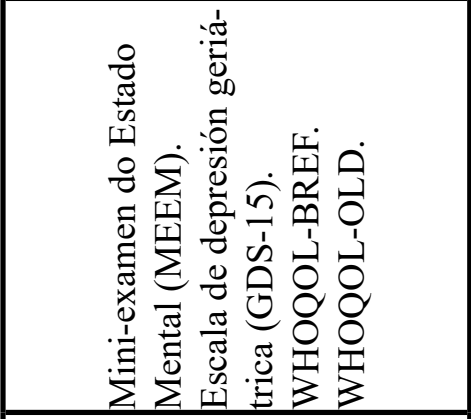 & 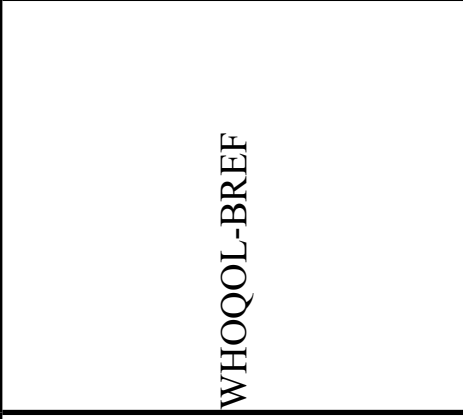 & 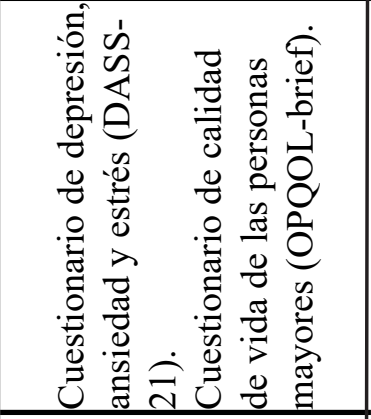 \\
\hline 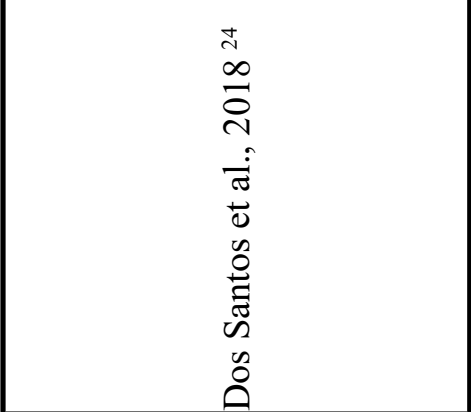 & 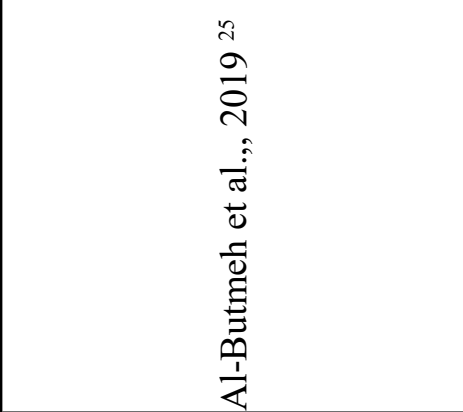 & 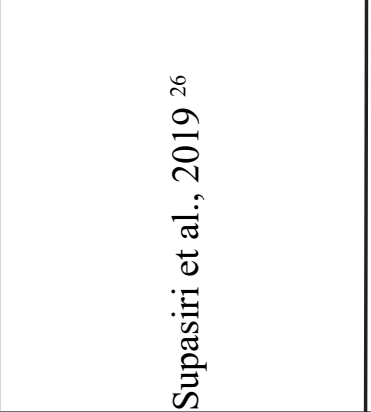 \\
\hline
\end{tabular}




\section{Resultados}

Un total de 1.231 estudios fueron identificados al aplicar la estrategia de búsqueda en las bases de datos Medline, LILACS, CINAHL y Scielo. La trayectoria de identificación, selección, elegibilidad e inclusión de los estudios seleccionados se puede visualizar en la figura 1.

Los artículos incluidos para su análisis en el estudio son 15 12-26 (Tabla 1 y 2). La mayoría de los artículos 7 (46.6\%) fueron obtenidos desde la base de datos bibliográfica MEDLINE 12,14-16, 18, 25,26 y el inglés fue el idioma predominante $7(46.6 \%) 12,14$ 16, 18, 25,26. En América Latina se realizaron la mayoría de los estudios 8 (53.3\%), en países como Colombia, Brasil y México 13,17,19, 20-24. La mayor parte de los artículos fueron publicados entre los años 2017 y 2019 (8, 53.3\%) 19-26. El tipo de estudio fue transversal descriptivo y/o correlacional en la totalidad de los casos y solo uno fue transversal comparativo entre dos periodos de tiempo 16.

Con respecto a las características particulares de los sujetos de estudio, la población total fue de 13.589 adultos mayores, siendo en su mayoría mujeres. La menor muestra estuvo compuesta por 50 participantes $22 \mathrm{y}$ la mayor por 4.248 adultos mayores 16 .

La edad de los participantes varió desde 55 a 112 años y el promedio de edad se encontró en el rango de 65 a 75 años. Solo un estudio incluyó a personas a partir de 55 años 18 , pues en su mayoría abarcan a sujetos a partir de los 60 años. Ocho artículos refieren que en sus criterios de selección para participar del estudio se excluyeron a los adultos mayores con déficit cognitivo evaluado por las escalas Mini Examen Cognoscitivo (MMSE), Test de valoración mental Pfeiffer y Miniexamen do Estado Mental (MEEM) 13,15,17,20-24.

Los instrumentos relativos a las mediciones de la calidad de vida fueron, principal- mente, WHOQOL-BREF 12,13,15,20,23-25 (7 estudios), Geriatric depression scale GDS 12,18,23,24 (4 estudios), WHOQOL-OLD 19,23,24 (3 estudios), SF-12 14,16 (2 estudios) y el Cuestionario de salud general de Goldberg GHQ12 13,22 (2 estudios).

Para la medición de la salud mental, los instrumentos más utilizados fueron la Escala de depresión geriátrica GDS 12,18,23,24 y la Escala de valoración geriátrica de depresión de Yesavage 17,21. Se utilizó, en promedio para cada estudio, 2 escalas de medición, una para calidad de vida y otra para salud mental.

\section{Discusión}

La revisión de la literatura analizada evidencia que la discapacidad mental y el deterioro de la calidad de vida afecta, actualmente a los adultos mayores en las diversas regiones del mundo como un impactante problema de salud pública 1-4,5.

Sobre la base de la literatura analizada, se logra identificar que los temas salud mental y calidad de vida de los adultos mayores es de un interés a investigar más bien reciente, evidenciando por una mayor cantidad de publicaciones en los 3 últimos años 19-26.

La totalidad de los estudios incluidos fueron transversales, lo que impide conocer la existencia de una relación causa-efecto, es decir, que la salud mental produzca un deterioro en la calidad de vida o lo contrario. Una de estas publicaciones realizó transversalmente una evaluación en 2 periodos, identificando cambios en el componente mental del cuestionario sobre calidad de salud, este componente disminuyo desde un $58.3 \%$ en el periodo $1997-2001$ a un $53.5 \%$ en 2008 2012, además aumentó al doble la prevalencia de los síntomas depresivos en este periodo de 7 años 16.

Cabe agregar que algunas investigaciones no son completamente comparables, debido a que algunas muestras fueron de tipo alea- 
Figura 1. Flujograma de identificación, selección, elegibilidad e inclusión de los estudios. Santiago Chile, 2020
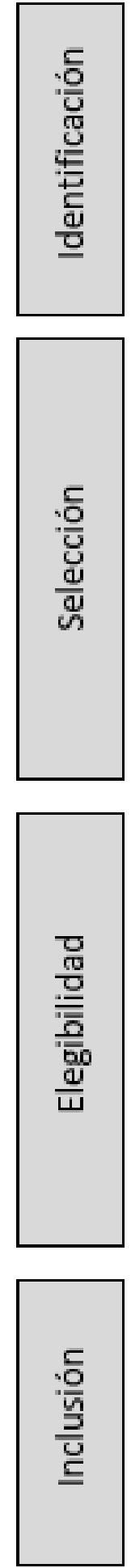

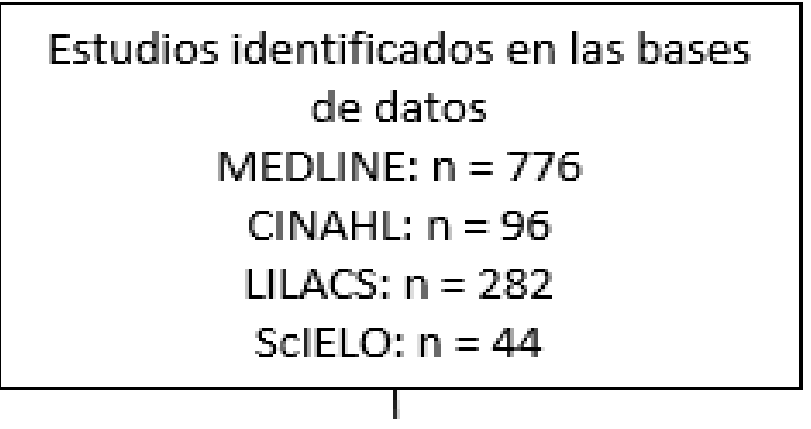

Aplicación de criterios de inclusión y exclusión

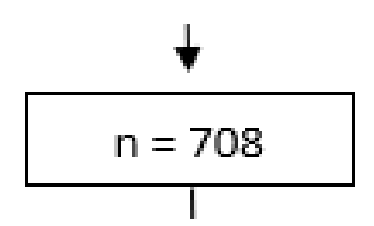

Registros duplicados

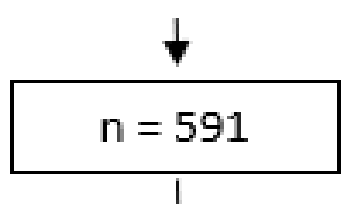

Lectura de títulos y resúmenes

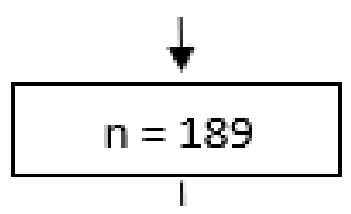

Lectura de texto completo

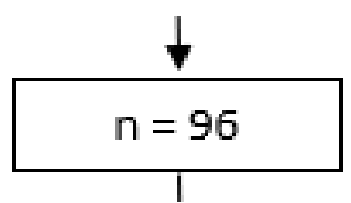

Evaluación de la calidad metodológica

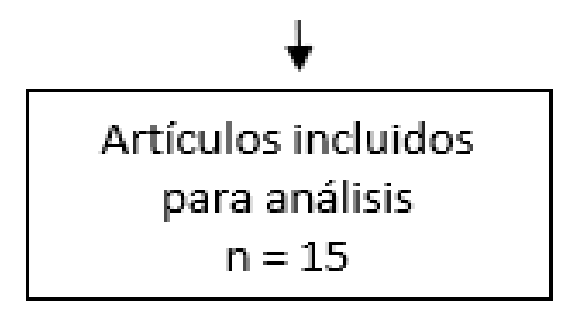


toria 18,26 , otras por conglomerados o estratificados 15,21 o no refieren datos completos sobre el tipo de muestra 25.

Con relación a los sujetos de estudio, el rango etario, en general, fue desde los 60 años, lo que está en concordancia con la definición de la OMS, que define a las personas de 60 y más años como personas de la "tercera edad". Esta definición incluye 3 rangos: las personas de 60 a 74 años llamadas de "edad avanzada", las de 75 a 90 denominadas "viejas" o "ancianas", y las que sobrepasan los 90 años que son las "longevas" 1,27. Esta clasificación es relevante, ya que en algunos estudios los resultados sobre la salud mental diferían de acuerdo al rango de edad 15,26.

Otra característica de la literatura analizada, fue la heterogeneidad de la población de estudio, que en algunos casos, los adultos mayores pertenecían a áreas rurales 14,15,26 $\mathrm{y}$ en otros, a programas de adultos mayores de población que residía en áreas urbanas 12. Estos últimos grupos tienen algún tipo de control de salud, redes de apoyo u otro beneficio, que se pueden considerar como variables que favorecen la calidad de vida

Los instrumentos utilizados para medir la calidad de vida en los adultos mayores fueron diversas escalas o cuestionarios que no permitieron, en su totalidad, una comparación entre los estudios, debido a sus diversos criterios y dimensiones a evaluar. El instrumento más utilizado para medir la calidad de vida fue World Health Organization Quality of Life Questionnaire en versión abreviada (WHOQOL-BREF), con 26 preguntas y que produce un perfil con respecto a calidad de vida en cuatro áreas: física, psicológica, relaciones sociales y ambiente, que según autores como Cardona-Arias et al., es el que presenta una excelente estructura conceptual y psicométrica, además de confiabilidad y adaptación cultural 28. Este instrumento ha sido validado en varios países e idiomas, por lo tanto, se presenta como una buena alter- nativa para uniformizar la evaluación de este tema 29.

Con relación a la salud mental, la Escala de depresión geriátrica GDS fue la más utilizada, evidenciando en 11 estudios que los porcentajes de depresión van desde un $5.7 \% 12$ hasta un $61.5 \% 23$. En la literatura analizada con la estrategia de búsqueda aplicada, se evidenció que las 2 patologías más prevalentes son la depresión y la ansiedad. Sin embargo, no se pesquisaron patologías relacionadas con la salud mental, tales como demencia o enfermedad de Alzheimer que también son comunes en la tercera edad.

Así, al relacionar esta incapacidad mental con la calidad de vida, existen 4 estudios que demostraron una asociación estadística entre el riesgo de depresión y el deterioro de la calidad de vida 17,20,23,24. Contrario a esto, el estudio de Supasiri et al., mostró que su población de estudio de adultos mayores tenía una calidad de vida media o alta, a pesar de presentar un $31.1 \%$ de ella trastornos mentales como estrés, depresión o ansiedad 26.

Algunos estudios mostraron las variables asociadas con una baja incidencia de sintomatología depresiva en estos adultos mayores, tales como convivir con pareja estable, contar con ingresos económicos, ser funcional para realizar actividades básicas de la vida diaria y percibir un adecuado apoyo social 21,26. Estas últimas evidencias científicas permiten guiar líneas de trabajo para ser desarrolladas con el objetivo de enfrentar este trastorno mental.

Por lo tanto, frente a la perspectiva del aceleramiento del envejecimiento poblacional, las evidencias científicas presentadas como revisiones sistemáticas, desempeñan un rol fundamental con respecto al aporte que hacen las ciencias, ya que, identifican las dimensiones de riesgo, además de las protectoras en relación con el manejo de este ámbito con el objetivo de enfrentar este gran desafío mundial. 


\begin{abstract}
Resumen
Introducción: Actualmente el número de personas con más de 60 años es el segmento etario que más crece a nivel mundial, lo que representa un gran desafio para la salud pública. Objetivo: Identificar a través de evidencias científicas, la existencia de una relación entre los trastornos de salud mental y la calidad de vida de los adultos mayores. Método: La investigación fue realizada mediante una revisión sistemática, en enero de 2020, en las bases bibliografías MEDLINE, LILACS, CINAHL y ScIELO, utilizando los descriptores "aged", "elderly", "aged,80 and over", "mental health", "dementia”, “depression", “alzheimer disease", "quality of life”, “WHOQOL-BREE”, “WHOQOL-BREF”, “WHOQOL-OLD”, "SF-12”, "SF-36", “NdF”, "geriatric depression scale" $y$ "mental health scales". Resultados: De los 1.231 artículos localizados, 15 fueron incluidos en el análisis. En 4 estudios se demostró una asociación estadística entre el riesgo de depresión y el deterioro de la calidad de vida. No obstante, un estudio mostró que la población de adultos mayores tenía una calidad de vida definida como media o alta, a pesar de presentar un 31.1\% de esta población trastornos mentales como estrés, depresión o ansiedad. Conclusiones: Para evaluar la calidad de vida y salud mental se evidencia un aumento de publicaciones en los últimos 3 años. Los instrumentos utilizados fueron diversas escalas o cuestionarios. Se identificaron varios factores que afectan positivamente o negativamente la relación entre la salud mental y la calidad de vida de los adultos mayores.

Palabras clave: Anciano; Salud Mental; Calidad de Vida
\end{abstract}

\section{Referencias Bibliográficas}

1.- World Health Organization (WHO). World report on ageing and health. Luxembourg: WHO; 2015. Pág.282. Disponible:http://apps.who.int/iris/bitstr0665/186463/1/9789240694811_ eng.pdf

2.- Departamento de Salud Mental y Abuso de Sustancias. Invertir en salud mental. Organización Mundial de la Salud, Ginebra. 2004. Pág. 52. Disponible: https://www.who.int/mental health/advocacy/en/spanish_final.pdf

3.- Jané-Llopis E, Gabilondo A. La salud mental de las personas mayores: Documento de consenso. Luxembourg. Comisión Europea. 2008. Pág. 35. Disponible: http://www.ec-mental-health. process.net
4.- $\quad$ Lang G, Resch K, Hofer K, Braddick F, Gabilondo A. Background document for the Thematic Conference on Mental Health and Well-being among Older. Luxembourg: European Communities. European Communities, 2010. Pág. 68. Disponible: http://ec.europa. eu/health/mental_health/policy/index en.htm

5.- Asociación Guipuzcoana de Familiares y Personas con Problemas de Salud Mental - AGIFES. Nueva definición de salud mental. Disponible: https:// agifes.org/es/campanas/salud-mental-para-todos/nueva-definicion-salud-mental

6.- Secker, J. Mental Health Promotion Theory and Practice: Implications for Implementation of Our Healthier Nation. MHRJ 1998;3(2):5-12. https:// 
doi.org/10.1108/13619322199800009

7.- Urzúa M, Caqueo-Urízar A. Calidad de vida: Una revisión teórica del concepto. Ter Psicol. 2012;30(1):6171. http://dx.doi.org/10.4067/S071848082012000100006

8.- Urzúa A. Calidad de vida relacionada con la salud: Elementos conceptuales. Rev Med Chile 2010;138(3):358365. http://dx.doi.org/10.4067/S003498872010000300017

9.- Barbosa FT, Lira AB, Oliveira Neto OB, Santos LL, Santos IO, Barbosa LT, et al. [Tutorial for performing systematic review and meta-analysis with interventional anesthesia studies]. Rev Bras Anestesiol. 2019 May - Jun;69(3):299-306. doi: 10.1016/j. bjan.2018.11.007.

10.- Higgins JPT, Green S (editors). Cochrane Handbook for Systematic Reviews of Interventions Version 5.1.0 [updated March 2011]. The Cochrane Collaboration, 2011. Disponible: www.cochrane-handbook.org.

11.- Moher D, Shamseer L, Clarke M, Ghersi D, Liberati A, Petticrew M, et al. Preferred reporting items for systematic review and meta-analysis protocols (PRISMA-P) 2015 statement. Syst Rev. 2015 Jan 1;4:1. doi: 10.1186/2046-4053-4-1.

12.- Kosulwit L. Mental health status, including depression and quality of life among members of an elderly club in suburban Bangkok. J Med Assoc Thai. 2012 Jan;95 Suppl 1:S92-101.

13.- Flores M, Colunga C, González M, Vega M, Cervantes G, Valle $M$ et al. Salud mental y calidad de vida en adultos mayores. Uaricha 2013;10(21):113. Disponible: file://D:/Usuario/ Downloads/Salud_mental_y_calidad_ de_vida_en_adultos_mayores_Mental_health_and_quality_of_life_in_ the_elderly.pdf
14.- Liang Y, Wu W. Exploratory analysis of health-related quality of life among the empty-nest elderly in rural China: an empirical study in three economically developed cities in eastern China. Health Qual Life Outcomes. 2014 Apr 25;12:59. doi: 10.1186/1477-7525-1259.

15.- Xie JF, Ding SQ, Zhong ZQ, Yi QF, Zeng SN, Hu JH, et al. La salud mental es el factor más importante que influye en la calidad de vida en los adultos mayores que se quedan cuando las familias emigran fuera de las zonas rurales de China. Rev.Latino-Am. Enfermagem. 2014;22(3):364-70. doi: 10.1590/0104-1169.3400.2425

16.- Baumeister SE, Schomerus G, Schmidt CO, Möckel F, van den Berg N, Hoffmann $\mathrm{W}$, et al. Change in depressive symptoms and mental health-related quality of life in northeast Germany between 1997-2001 and 2008-2012. Int J Public Health. 2015 Jan;60(1):339. doi: $10.1007 / \mathrm{s} 00038-014-0632-0$

17.- Segura-Cardona A, Cardona-Arango D, Segura-Cardona A, Garzón-Duque M. Riesgo de depresión y factores asociados en adultos mayores. Antioquia, Colombia. 2012. Rev. Salud Pública. 2015;17(2):184-194. DOI: http://dx. doi.org/10.15446/rsap.v17n2.41295

18.- Keshavarzi S, Ahmadi SM, Lankarani KB. The impact of depression and malnutrition on health-related quality of life among the elderly Iranians. Glob J Health Sci. 2014 Nov 26;7(3):161-70. doi: 10.5539/gjhs.v7n3p161.

19. Acosta C, Tánori J, García R, Echeverría S, Vales J, Rubio L. Soledad, depresión y calidad de vida en adultos mayores mexicanos. Psicología y Salud. 2017;27(2):179-188. Disponible: http://psicologiaysalud.uv.mx/index. $\mathrm{php} / \mathrm{psicysalud} /$ article/view/2535.

20.- Soares S, Silva P, Santos J, Silva L. 
Associação entre depressão e qualidade de vida em idosos: atenção primária à saúde. Enferm. UFRJ. 2017;25, e19987. Disponible: http://dx.doi. org/10.12957/reuerj.2017.19987.

21.- Vargas-Ricardo S, Melquizo-Herrera E. Calidad de vida en adultos mayores en Cartagena, Colombia. Rev. Salud Pública. 2017;19(4):549-554. Disponible: http://www.scielo.org.co/pdf/rsap/ v19n4/0124-0064-rsap-19-04-00549. pdf

22.- Quintero M, Cerquera A. Estado de salud general y perfil biopsicosocial de adultos mayores sanos o excepcionales de Santander, Colombia. Perspect. Psicol. 2018;14(1):109-120. Disponible: http://www.scielo.org.co/pdf/dpp/ v14n1/1794-9998-dpp-14-01-00109. pdf

23.- Gato J M, Zenevicz LT, Faganello Madureira VS, Gaffuri da Silva T, Sedrez Celich KL, Silva de Souza S, et al. Av Enferm. 2018;36(3) 302-310. doi: 10.15446/av.enferm.v36n3.68498

24.- Dos Santos V, Souza R, Cruz G, Santos Í, Cassotti C. Qualidade de vida e depressão em domicílios no contexto doméstico. Enfermería. Actual de Costa Rica 2018;(34):53-66.Disponible: http://dx.doi.org/10.15517/revenf. v0i34.30983.

25.- Al-Butmeh S, Al-Khataib N. Mental health and quality of life of elderly people in the Bethlehem district: a cross-sectional study. Lancet. 2018 Feb 21;391 Suppl 2:S46. doi: 10.1016/ S0140-6736(18)30412-4.

26.- Supasiri T, Lertmaharit S, Rattananupong T, Kitidumrongsuk P, Lohsoonthorn V. Mental health status and quality of life of the elderly in rural Saraburi. Chula Med J. 2019;63(2):95101. doi:10.14456/clmj.1476.14

27.- World Health Organization. WHOQOL-BREF Introduction, administra- tion, scoring and generic version of the assessment. Geneva: WHO; 1996. Disponible: https://www.who.int/mental_health/media/en/76.pdf

28.- Cardona-Arias J, Higuita-Gutiérrez L. Aplicaciones de un instrumento diseñado por la OMS para la evaluación de la calidad de vida. $\mathrm{Rev} \mathrm{Cu}-$ bana Salud Pública. 2014;40 (2):175189. Disponible: http://scielo.sld.cu/ scielo.php?script=sci_arttext\&pi$\mathrm{d}=$ S0864-34662014000200003\&lan$\mathrm{g}=\mathrm{es}$

29. Espinoza I, Osorio P, Torrejón MJ, Lucas-Carrasco R, Bunout D. Validación del cuestionario de calidad de vida (WHOQOL-BREF) en adultos mayores chilenos. Rev méd Chile 2011;139(5):579-586. Disponible: http://dx.doi.org/10.4067/ S0034-98872011000500003.

Correspondencia a:

Fanny López Alegría.

Escuela de Enfermería, Universidad Andrés Bello. Republica 217, Santiago, Chile.

(56-2) 27703516 - 984729215

fanny.lopez@unab.cl 